Article

\title{
On the Partition of Energies for the Backward in Time Problem of Thermoelastic Materials with a Dipolar Structure
}

\author{
M. Marin ${ }^{1, *(D)}$, S. Vlase ${ }^{2}$, R. Ellahi ${ }^{3,4}$ and M.M. Bhatti ${ }^{5,6}$ \\ 1 Department of Mathematics and Computer Science, Transilvania University of Brasov, \\ 500093 Brasov, Romania \\ 2 Department of Mechanical Engineering, Transilvania University of Brasov, 500093 Brasov, Romania \\ 3 Center for Modeling \& Computer Simulation, Research Institute, King Fahd University of Petroleum \& \\ Minerals, Dhahran 31261, Saudi Arabia \\ 4 Department of Mathematics \& Statistics, Faculty of Basic and Applied Sciences (FBAS), International Islamic \\ University (IIUI), Islamabad 44000, Pakistan \\ 5 College of Mathematics and Systems Science, Shandong University of Science and Technology, \\ Qingdao 266590, Shandong, China \\ 6 Shanghai Institute of Applied Mathematics and Mechanics, Shanghai University, Shanghai 200072, China \\ * Correspondence: m.marin@unitbv.ro
}

Received: 12 June 2019; Accepted: 26 June 2019; Published: 2 July 2019

\begin{abstract}
We first formulate the mixed backward in time problem in the context of thermoelasticity for dipolar materials. To prove the consistency of this mixed problem, our first main result is regarding the uniqueness of the solution for this problem. This is obtained based on some auxiliary results, namely, four integral identities. The second main result is regarding the temporal behavior of our thermoelastic body with a dipolar structure. This behavior is studied by means of some relations on a partition of various parts of the energy associated to the solution of the problem.
\end{abstract}

Keywords: backward in time problem; dipolar thermoelastic body; uniqueness of solution; Cesaro means; partition of energies

\section{Introduction}

In our study, we approach a thermoelastic body having a dipolar structure. This kind of structure falls within a more general theory, namely, the theory of bodies with microstructure. The first studies in this context were published by Eringen (see, for instance, references [1,2]). One may deduce the importance of the dipolar structure due to the large number of published studies dedicated to this topic, of which we can mention [3-7]. As such, our present work can be considered a continuation in this respect.

A continuation of the theories of microstructure, is a theory that takes into account the voids in the materials. It is considered that the initiators of this theory were Nunziato and Cowin, in their known paper [8]. After that, the number of studies within this topic has grown impressively. We want to enumerate some of these [9-16]: The first result for the backward in time problem belongs to Serrin, who approached this problem in the context of Navier-Stokes equations (see [17]). In the paper [17], we find some uniqueness in the results with regards to the forward in time problem. After that, the number of studies dedicated to the backward in time problem has increased considerably. Of particular importance are the works [18-27]. We have to point out that the results obtained by Ciarletta in [23], and Ciarletta and Chirita in [24] were improved by Quintanilla in [25]. In addition, Quintanilla approached the question of location in time for solutions to the backward in time problem, 
in the context of thermoelasticity of Green and Naghdi [26,27] and the theory of porous thermoelastic bodies. The elastic porous bodies were also approached by Iovane and Passarella in [28]. The forward in time problem, in the context of theory for thermomicrostretch elastic solids, was approached by Passarella and Tibullo in [29]. It is worth noting that the idea of considering non-standard problems, in the context of the general theory of bodies having a dipolar structure, was inspired by Quintanilla and Straughan's work [30]. In [31,32] it is proved that the nonhomogenous temperature field has a profound influence on the nanobeam mechanics. Additionally, [33] is a recent contribution on stress-driven nonlocal modeling of thermoelastic nanostructures.

Here is the plane of our study. First of all, we summarize the main equations, the initial conditions, and the boundary data of the mixed problem. Then, we prove some estimates for the gradient of classical and dipolar displacements, and for the gradient of the function of voids. In the last part of our study we prove the main result, namely, the continuous dependence of solutions-with regards to the coefficients that couple the equations describing the dipolar deformation-with the equations that describe the behavior of voids. The description of the continuous dependence was possible due to the definition of an adequate measure.

\section{Basic Equations and Conditions}

In our paper, we approach a thermoelastic body having a dipolar structure. We will use an anisotropic body, which is situated in a regular domain $D$, included in the physical space $E^{3}$, that is, the three-dimensional Euclidean space. Consider that the boundary of the domain is a piecewise smooth surface $\partial D$. The closure of $D$ is usual denoted by $\bar{D}, \bar{D}=D \cup \partial D$. An orthonormal system of references is introduced, and then tensors and vectors have components with Latin subscripts over $1,2,3$. Typical conventions for summation over repeated indices and for derivation operations are implied. So, a subscript preceded by a comma is for a partial derivative with regards to corresponding spatial coordinate; while a superposed dot is for a derivative with regards to time variable. All the functions we use are assumed to be sufficiently regular as necessary. Additionally, if there is no possibility of confusion, then the dependence of function with regards to its spatial or time variables will be omitted. The evolution of the body with a dipolar structure will be described with the help of the following specific variables:

$$
\mathrm{u}_{i}(x, t), \phi_{i j}(x, t), \theta(x, t),(x, t) \in D \times\left[0, t_{0}\right) .
$$

Here, we denoted by $\mathrm{u}_{i}$ the components of the displacement vector field, by $\phi_{i j}$ the components of the dipolar displacement tensor field, and by $\theta$ the absolute temperature.

Using the above variables $\mathrm{u}_{i}(x, t)$, and $\phi_{i j}(x, t)$ we will introduce the components of the tensors of strain, namely, $\varepsilon_{i j}, \kappa_{i j}$, and $\chi_{i j k}$, as follows:

$$
2 \varepsilon_{i j}=u_{j, i}+u_{i, j}, \kappa_{i j}=u_{j, i}-\phi_{i j}, \chi_{i j k}=\phi_{i j, k} .
$$

All our considerations are made within a linear theory, therefore it is natural to consider that the Helmholtz's free energy is a quadratic form with regards to its independent constitutive variables. The Helmholtz's free energy in the reference configuration will be denoted by $W$. So, in accordance with the principle of conservation of energy, we develop in series the function $W$ and we keep the terms only until the second order. Because the reference state was assumed to be free of loadings, we deduce that the Helmholtz's free energy per mass can be considered of the following form (see [30]):

$$
\begin{aligned}
& W=\frac{1}{2} A_{i j m n} \varepsilon_{i j} \varepsilon_{m n}+D_{i j m n} \varepsilon_{i j} \kappa_{m n}+F_{i j m n r} \varepsilon_{i j} \chi_{m n r}+\frac{1}{2} B_{i j m n} \kappa_{i j} \kappa_{m n} \\
& +G_{i j m n r} \kappa_{i j} \chi_{m n r}+\frac{1}{2} C_{i j k m n r} \chi_{i j k} \chi_{m n r}-a_{i j} \varepsilon_{i j} \theta-b_{i j} \kappa_{i j} \theta-c_{i j k} \chi_{i j k} \theta-\frac{1}{2} c \theta^{2} .
\end{aligned}
$$

We will use this form of free energy used in the entropy production inequality and deduce the motion equations. In addition, from the same inequality, the constitutive equations are obtained. These 
equations express the tensors of stress with the help of the tensors of deformation. We will denote the components of the stress measures by $\tau_{i j}, \eta_{i j}$, and $\mu_{i j k}$. In this way, the constitutive equations establish a connection between the tensors $\tau_{i j}, \eta_{i j}, \mu_{i j k}$ and the tensors $\varepsilon_{i j}, \kappa_{i j}, \chi_{i j k}$.

We will use a procedure similar to that used by Green and Rivlin in [6], so that considering the Helmholtz' free energy (3) we deduce the next constitutive equations

$$
\begin{aligned}
& \tau_{i j}=\frac{\partial W}{\partial \varepsilon_{i j}}=A_{i j m n} \varepsilon_{m n}+D_{m n i j} \kappa_{m n}+F_{m n r i j} \chi_{m n r}-a_{i j} \theta, \\
& \eta_{i j}=\frac{\partial W}{\partial \kappa_{i j}}=D_{i j m n} \varepsilon_{m n}+B_{i j m n} \kappa_{m n}+G_{i j m n r} \chi_{m n r}-b_{i j} \theta, \\
& \mu_{i j k}=\frac{\partial W}{\partial \chi_{i j k}}=F_{i j k m n} \varepsilon_{m n}+G_{m n i j k} \kappa_{m n}+C_{i j k m n r} \chi_{m n r}-c_{i j k} \theta, \\
& \eta=-\frac{\partial W}{\partial \theta}=a_{i j} \varepsilon_{i j}+b_{i j} \kappa_{i j}+c_{i j k} \chi_{i j k}+c \theta,
\end{aligned}
$$

which are satisfied in $D \times\left[0, t_{0}\right)$. Here, we denoted by $\eta$ the entropy per unit mass.

For the vector of heat flux, having the components $q_{i}$ we have a classical constitutive relation, namely,

$$
q_{i}=K_{i j} \theta_{j}
$$

where $K_{i j}$ is the thermal conductivity symmetric tensor.

Also, we can deduce the main equations that govern the thermoelasticity of bodies with a dipolar structure, namely (see $[5,6])$ :

- the motion equations:

$$
\begin{aligned}
& \left(\tau_{i j}+\eta_{i j}\right)_{, j}+\rho f_{i}=\rho \ddot{\mathrm{u}}_{i} \\
& \mu_{i j k, i}+\eta_{j k}+\rho g_{j k}=I_{k r} \ddot{\phi}_{j r}
\end{aligned}
$$

- the equation of energy:

$$
\rho T_{0} \dot{\eta}=q_{i, i}+\rho r
$$

The signification of the notations that we introduced in preceding equations is as follows: $\rho$, the density of mass, which is a constant; $I_{i j}$, the symmetric tensor of microinertia; $k$, the intrinsic inertia; $\varepsilon_{i j}, \kappa_{i j}, \chi_{i j k}$, the strain tensors; $\tau_{i j}, \eta_{i j}, \mu_{i j k}$, the stress tensors; $f_{i}$, the body forces; $g_{j k}$, the dipolar charges; $A_{i j m n}, B_{i j m n}, \ldots, a_{i j}$, the functions that describe the properties of the material in terms of elasticity. Suppose the following symmetry relations take place:

$$
\begin{aligned}
& A_{i j m n}=A_{j i m n}=A_{m n i j}, B_{i j m n}=B_{m n i j}, a_{i j}=a_{j i}, \\
& C_{i j k m n r}=C_{m n r i j k}, F_{i j k m n}=F_{i j k n m}, D_{i j m n}=D_{i j n m} .
\end{aligned}
$$

Assuming that there are no supply terms and taking into account the constitutive Equations (4) and (5) and the kinematic Equation (2), Equations (6) and (7) become

$$
\begin{array}{r}
\rho \ddot{\mathrm{u}}_{i}=\left[\left(C_{i j m n}+G_{i j m n}\right) \mathrm{u}_{n, m}+\left(G_{m n i j}+B_{i j m n}\right)\left(\mathrm{u}_{n, m}-\phi_{m n}\right)+\right. \\
\left.+\left(F_{m n r i j}+D_{i j m n r}\right) \phi_{n r, m}-\left(a_{i j}+b_{i j}\right) \theta\right]_{, j}, \\
I_{k r} \ddot{\phi}_{j r}=\left[F_{i j k m n} \mathrm{u}_{n, m}+D_{m n i j k}\left(\mathrm{u}_{n, m}-\phi_{m n}\right)+A_{i j k m n r} \phi_{n r, m}-c_{i j k} \theta\right]_{, i}+ \\
+G_{j k m n} \mathrm{u}_{m, n}+B_{j k m n}\left(\mathrm{u}_{n, m}-\phi_{m n}\right)+D_{j k m n r} \phi_{n r, m}-b_{j k} \theta, \\
K_{i j}\left(\theta_{, j}\right)_{, i}=-T_{0}\left[a_{i j} \dot{\mathrm{u}}_{i, j}+b_{i j}\left(\dot{\mathrm{u}}_{j, i}-\dot{\phi}_{i j}\right)+c_{i j k} \dot{\phi}_{i j, k}+c \dot{\theta}\right] .
\end{array}
$$

From now, we will assume that the Equations (2), (4), and (9) will be satisfied on the interval $(-\infty, 0]$. 
The outward unit normal to the surface $\partial D$ has the components $n_{i}$. With the help of this normal we can define the surface traction's of components $t_{i}$, the surface couple of components $\mu_{j k}$, and the flux of heat, $q$. All of this makes sense in every point of regularity of the boundary $\partial D$ and has the following expressions

$$
t_{i}=\left(\tau_{i j}+\eta_{i j}\right) n_{j}, \mu_{j k}=\mu_{i j k} n_{i}, q=q_{i} n_{i} .
$$

In close relation to these surface tractions, we consider the following homogeneous boundary conditions:

$$
\begin{aligned}
& \mathrm{u}_{i}(x, t)=0,(x, t) \in \partial D_{u} \times(-\infty, 0], t_{i}=0,(x, t) \in \partial D_{u}^{c} \times(-\infty, 0], \\
& \phi_{i j}(x, t)=0,(x, t) \in \partial D_{\phi} \times(-\infty, 0], m_{j k}=0,(x, t) \in \partial D_{\phi}^{c} \times(-\infty, 0], \\
& \theta(x, t)=0,(x, t) \in \partial D_{\theta} \times(-\infty, 0], q=0,(x, t) \in \partial D_{\theta}^{c} \times(-\infty, 0],
\end{aligned}
$$

where the surfaces $\partial D_{u}, \partial D_{\phi}, \partial D_{\theta}$, and its complements $\partial D_{u}^{c}, \partial D_{\phi}^{c}, \partial D_{\theta}^{c}$ are subsurfaces of the border $\partial D$, which are subject to the following restrictions:

$$
\begin{gathered}
\partial \bar{D}_{u} \cup \partial D_{u}^{c}=\partial \bar{D}_{\phi} \cup \partial D_{\phi}^{c}=\partial \bar{D}_{\theta} \cup \partial D_{\theta}^{c}=\partial D, \\
\partial D_{u} \cap \partial D_{u}^{c}=\partial D_{\phi} \cap \partial D_{\phi}^{c}=\partial D_{\theta} \cap \partial D_{\theta}^{c}=\varnothing .
\end{gathered}
$$

We still have to add the final restrictions. So, on the closed domain $\bar{D}$ we have:

$$
\begin{aligned}
& \mathrm{u}_{i}(x, 0)=\mathrm{u}_{i}^{0}(x), \dot{\mathrm{u}}_{i}(x, 0)=\mathrm{u}_{i}^{1}(x), \theta(x, 0)=\theta^{0}(x), \\
& \phi_{i j}(x, 0)=\phi_{i j}^{0}(x), \dot{\phi}_{i j}(x, 0)=\phi_{i j}^{1}(x),
\end{aligned}
$$

where $\mathrm{u}_{i}^{0}(x), \mathrm{u}_{i}^{1}(x), \phi_{i j}^{0}(x), \phi_{i j}^{1}(x)$, and $\theta^{0}(x)$ are continuous prescribed functions in all points where they are defined. Additionally, these functions are assumed be compatible with conditions (11) on the appropriate subsets of $\partial D$.

Let us consider the internal energy density $\Psi$ (see [30]), which has the following expression:

$$
\begin{aligned}
& \Psi=\frac{1}{2} A_{i j m n} \varepsilon_{i j} \varepsilon_{m n}+D_{i j m n} \varepsilon_{i j} \kappa_{m n}+F_{i j m n r} \varepsilon_{i j} \chi_{m n r}+ \\
& +\frac{1}{2} B_{i j m n} \kappa_{i j} \kappa_{m n}+G_{i j m n r} \kappa_{i j} \chi_{m n r}+\frac{1}{2} C_{i j k m n r} \chi_{i j k} \chi_{m n r} .
\end{aligned}
$$

$\mathcal{P}$ is denoted the so-called boundary-final value problem, which consists of Equation (9), the boundary restrictions (11), and the final data (12).

To obtain the results we have proposed, we will have to impose some conditions on the functions we are dealing with.

So, if $J_{m}(x)$ is the minimum eigenvalue of the inertia tensor $I_{i j}(x)$, then we need to assume that $J_{m}$ and $\rho$ are continuous functions and the constitutive coefficients are of class $C^{1}(D)$. We also assume that:

(a) $\rho(x) \geq a_{1}, J_{m}(x) \geq a_{2}, c(x) \geq c_{0}$, where $a_{1}, a_{2}, c_{0}$ are real positive constants;

(b) the tensor $K_{i j}$ is positive definite;

(c) the internal energy density $\Psi$ is a positive definite quadratic form.

Based on hypothesis (b), we deduce that there exist two positive numbers, $K_{m}$ and $K_{M}$, so that

$$
K_{m} \theta_{, i} \theta_{, j} \leq K_{i j} \theta_{, i} \theta_{, j} \leq K_{M} \theta_{, i} \theta_{, j}
$$

and, as a consequence of the hypothesis (c), we can find the positive constants $M_{1}$ and $M_{2}$ so that the next inequality is satisfied:

$$
\frac{M_{1}}{2}\left(\varepsilon_{i j} \varepsilon_{i j}+\kappa_{i j} \kappa_{i j}+\chi_{i j k} \chi_{i j k}\right) \leq \Psi \leq \frac{M_{2}}{2}\left(\varepsilon_{i j} \varepsilon_{i j}+\kappa_{i j} \kappa_{i j}+\chi_{i j k} \chi_{i j k}\right) .
$$


These hypotheses are not considered as very restrictive, as they are commonly imposed in mechanics of continuous media.

It is not difficult to equate our boundary-final value problem $\mathcal{P}$ with a boundary-initial problem, denoted by $\mathcal{P}^{\prime}$, by a convenient change of variables. In this regard, we set $h^{\prime}\left(t^{\prime}\right)=h(t)$, for $t^{\prime}=-t$. But, to simplify writing, we will give up the sign "prime" so that the $\mathcal{P}^{\prime}$ problem will be defined by the following conditions and equations:

- the motion Equations (9a) and (9b), satisfied in $D \times[0, \infty)$;

- the energy equation:

$$
K_{i j}\left(\theta_{, j}\right)_{, i}=T_{0}\left[a_{i j} \dot{\mathrm{u}}_{i, j}+b_{i j}\left(\dot{\mathrm{u}}_{j, i}-\dot{\phi}_{i j}\right)+c_{i j k} \dot{\phi}_{i j, k}+c \dot{\theta}\right], \text { in } D \times[0, \infty) ;
$$

- the geometric Equation (2), satisfied in $D \times[0, \infty)$;

- the constitutive Equation (4), satisfied in $D \times[0, \infty)$;

- the initial conditions (11), satisfied in $\bar{D}$;

- the boundary conditions:

$$
\begin{aligned}
& \mathrm{u}_{i}(x, t)=0,(x, t) \in \partial D_{u} \times[0, \infty), t_{i}=0,(x, t) \in \partial D_{u}^{c} \times[0, \infty), \\
& \phi_{i j}(x, t)=0,(x, t) \in \partial D_{\phi} \times[0, \infty), m_{j k}=0,(x, t) \in \partial D_{\phi}^{c} \times[0, \infty), \\
& \theta(x, t)=0,(x, t) \in \partial D_{\theta} \times[0, \infty), q=0,(x, t) \in \partial D_{\theta}^{c} \times[0, \infty) .
\end{aligned}
$$

\section{Main Result}

We first establish some integral identities regarding a solution $\mathbf{u}=\left(\mathrm{u}_{i}, \phi_{i j}, \theta\right)$ of the mixed problem $\mathcal{P}^{\prime}$. These will be useful in obtaining the important results of our study.

Proposition 1. If the ordered array $\mathbf{u}=\left(\mathrm{u}_{i}, \phi_{i j}, \theta\right)$ satisfies the mixed problem $\mathcal{P}^{\prime}$, then the following equality takes place:

$$
\begin{gathered}
\int_{B}\left[\frac{1}{2}\left(\rho \dot{\mathrm{u}}_{i}(t) \dot{\mathrm{u}}_{i}(t)+I_{j k} \dot{\phi}_{j m}(t) \dot{\phi}_{k m}(t)\right)+\Psi(t)+\frac{1}{2} c \theta^{2}(t)\right] d V= \\
=\int_{B}\left[\rho \dot{\mathrm{u}}_{i}(0) \dot{\mathrm{u}}_{i}(0)+I_{j k} \dot{\phi}_{j m}(0) \dot{\phi}_{k m}(0)+\Psi(0)+\frac{1}{2} c \theta^{2}(0)\right] d V+ \\
\quad+\int_{0}^{t} \int_{D} \frac{1}{T_{0}} K_{i j} \theta_{, i}(\tau) \theta_{, j}(\tau) d V d \tau, \forall t \in[0, \infty) .
\end{gathered}
$$

Proof. Taking into account the kinematics compatibility relations (2) and the differential conditions of equilibrium (9a) and (9b), we obtain the following equality:

$$
\begin{aligned}
& \frac{1}{2} \frac{\partial}{\partial t}\left(\rho \dot{\mathrm{u}}_{i}(t) \dot{\mathrm{u}}_{i}(t)+I_{j k} \dot{\phi}_{j m}(t) \dot{\phi}_{k m}(t)\right)= \\
& =\left[\left(\tau_{i j}+\eta_{i j}\right) \dot{\mathrm{u}}_{j}+\mu_{i j k} \dot{\phi}_{j k}\right]_{, i}-\left(\tau_{i j} \dot{\varepsilon}_{i j}+\eta_{i j} \dot{\kappa}_{i j}+\mu_{i j k} \dot{\chi}_{i j k}\right) .
\end{aligned}
$$

Taking into account the constitutive Equation (4), the symmetry relations (8), and the expression of the internal energy density $\Psi$ from (13), the last parentheses in the right-hand side of (19) becomes

$$
\left(\tau_{i j} \dot{\varepsilon}_{i j}+\eta_{i j} \dot{K}_{i j}+\mu_{i j k} \dot{\chi}_{i j k}\right)=\frac{\partial}{\partial t}\left(\Psi+\frac{1}{2} c \theta^{2}\right)+\left(\frac{1}{T_{0}} q_{j} \theta\right)_{, j}-\frac{1}{T_{0}} K_{i j} \theta_{, i} \theta_{, i} .
$$

We substitute Equation (20) into Equation (19), then the resulting equality is integrated on cylinder $[0, t] \times D$. If we use the theorem of divergence and consider the conditions to the limit (17), we are led to equality (18), such that the proof of the proposition is finished. 
In a similar way, one can demonstrate the identity that follows, as a complement to identity (18):

$$
\begin{aligned}
\int_{D}\left[\frac{1}{2}\left(\rho \dot{\mathrm{u}}_{i}(t) \dot{\mathrm{u}}_{i}(t)+I_{j k} \dot{\phi}_{j m}(t) \dot{\phi}_{k m}(t)\right)+\Psi(t)-\frac{1}{2} c \theta^{2}(t)\right] d V= \\
=\int_{D}\left[\rho \dot{\mathrm{u}}_{i}(0) \dot{\mathrm{u}}_{i}(0)+I_{j k} \dot{\phi}_{j m}(0) \dot{\phi}_{k m}(0)+\Psi(0)-\frac{1}{2} c \theta^{2}(0)\right] d V- \\
\quad-\int_{0}^{t} \int_{D}\left\{\dot{\mathrm{u}}_{i}(\tau)\left[\left(a_{i j}+b_{j i}\right) \theta(\tau)\right]_{, j}+\dot{\phi}_{i j}(\tau)\left[c_{i j k} \theta(\tau)\right]_{, k}-\right. \\
\left.\quad-b_{i j} \dot{\phi}_{i j}(\tau) \theta(\tau)+\frac{1}{T_{0}} K_{i j} \theta_{, i}(\tau) \theta_{, j}(\tau)\right\} d V d \tau, \forall t \in[0, \infty) .
\end{aligned}
$$

To simplify writing, we enter the notation

$$
\begin{aligned}
2 F(x, y)= & A_{i j m n} \varepsilon_{i j}(x) \varepsilon_{m n}(y)+D_{i j m n}\left[\varepsilon_{i j}(x) \kappa_{m n}(y)+\varepsilon_{i j}(y) \kappa_{m n}(x)\right]+ \\
+ & F_{i j m n r}\left[\varepsilon_{i j}(x) \chi_{m n r}(y)+\varepsilon_{i j}(y) \chi_{m n r}(x)\right]+B_{i j m n} \kappa_{i j}(x) \kappa_{m n}(y)+ \\
& +G_{i j m n r}\left[\kappa_{i j}(x) \chi_{m n r}(y)+\kappa_{i j}(y) \chi_{m n r}(x)\right]+C_{i j k m n r} \chi_{i j k}(x) \chi_{m n r}(y) .
\end{aligned}
$$

By using the symmetry relations (8), from (22) we deduce

$$
F(x, y)=F(y, x)
$$

By direct substitution in (22) and taking into account Equation (13), we also obtain

$$
F(\tau, \tau)=\Psi(\tau)
$$

Now, we can prove a result similar to (18), but in the case of homogeneous initial conditions.

Proposition 2. Consider a solution $\left(\mathrm{u}_{i}, \phi_{i j}, \theta\right)$ of the problem backward in time $\mathcal{P}^{\prime}$, which corresponds to null initial data. Then, the next identity takes place

$$
\begin{gathered}
\int_{B}\left[\frac{1}{2}\left(\rho \dot{\mathrm{u}}_{i}(t) \dot{\mathrm{u}}_{i}(t)+I_{j k} \dot{\phi}_{j m}(t) \dot{\phi}_{k m}(t)\right)-\frac{1}{2} c \theta^{2}(t)\right] d V= \\
=\int_{B}\left[\frac{1}{2} A_{i j m n} \varepsilon_{i j}(t) \varepsilon_{m n}(t)+D_{i j m n} \varepsilon_{i j}(t) \kappa_{m n}(t)+\right. \\
+F_{i j m n r} \varepsilon_{i j}(t) \chi_{m n r}(t)+\frac{1}{2} B_{i j m n} \kappa_{i j}(t) \kappa_{m n}(t)+ \\
\left.+G_{i j m n r} \kappa_{i j}(t) \chi_{m n r}(t)+\frac{1}{2} C_{i j k m n r} \chi_{i j k}(t) \chi_{m n r}(t)\right] d V,
\end{gathered}
$$

for all $t \in[0, \infty)$.

Proof. By direct calculations, for a fixed $t \in(0, \infty)$, we get the identity:

$$
\begin{array}{r}
\frac{\partial}{\partial t}\left(\rho \dot{\mathrm{u}}_{i}(\tau) \dot{\mathrm{u}}_{i}(2 t-\tau)+I_{j k} \dot{\phi}_{j m}(\tau) \dot{\phi}_{k m}(2 t-\tau)-c \theta(\tau) \theta(2 t-\tau)\right)= \\
=\rho \ddot{\mathrm{u}}_{i}(\tau) \dot{\mathrm{u}}_{i}(2 t-\tau)+I_{j k} \ddot{\phi}_{j m}(\tau) \dot{\phi}_{k m}(2 t-\tau)+c \theta(\tau) \dot{\theta}(2 t-\tau)- \\
\quad-\rho \dot{\mathrm{u}}_{i}(\tau) \ddot{\mathrm{u}}_{i}(2 t-\tau)+I_{j k} \dot{\phi}_{j m}(\tau) \ddot{\phi}_{k m}(2 t-\tau)-c \dot{\theta}(\tau) \theta(2 t-\tau) .
\end{array}
$$


Taking into account the kinematic Equation (2), the constitutive Equation (4), the motion Equation (9), and the symmetry relations (8), we are led to the equality

$$
\begin{gathered}
\frac{\partial}{\partial t}\left(\rho \dot{\mathrm{u}}_{i}(\tau) \dot{\mathrm{u}}_{i}(2 t-\tau)+I_{j k} \dot{\phi}_{j m}(\tau) \dot{\phi}_{k m}(2 t-\tau)-c \theta(\tau) \theta(2 t-\tau)\right)= \\
=\left[\left(\tau_{i j}+\eta_{i j}\right)(\tau) \dot{\mathrm{u}}_{i}(2 t-\tau)-\left(\tau_{i j}+\eta_{i j}\right)(2 t-\tau) \dot{\mathrm{u}}_{j}(\tau)+\right. \\
+\mu_{i j k} \ddot{\phi}_{j k}(\tau) \dot{\phi}_{j k}(2 t-\tau)-\mu_{i j k} \ddot{\phi}_{j k}(2 t-\tau) \dot{\phi}_{j k}(\tau)- \\
\left.\quad-\frac{1}{T_{0}} \theta(\tau) q_{i}(2 t-\tau)+\frac{1}{T_{0}} \theta(2 t-\tau) q_{i}(\tau)\right]_{, i}+F(\tau, 2 t-\tau),
\end{gathered}
$$

the function $F(.,$.$) being defined in (22).$

We just need to integrate this equality into $[0, t] \times D$, to keep in mind that the initial data are null and to use the definition (13), so we get the desired equality (25) and the proof of proposition is complete.

The following two propositions are also useful in establishing the main outcomes of our study.

Proposition 3. Consider that the ordered array $\mathbf{u}=\left(\mathrm{u}_{i}, \phi_{i j}, \theta\right)$ satisfies the mixed problem $\mathcal{P}^{\prime}$. Then, the following equality takes place

$$
\begin{gathered}
\int_{D}\left[\rho \mathrm{u}_{i}(t) \dot{\mathrm{u}}_{i}(t)+I_{j k} \phi_{j m}(t) \dot{\phi}_{k m}(t)-\frac{1}{2 T_{0}} K_{i j}\left(\int_{0}^{t} \theta(\tau) d \tau\right)_{, i}\left(\int_{0}^{t} \theta(\tau) d \tau\right)_{, j}\right] d V= \\
=\int_{D}\left[\rho \mathrm{u}_{i}(0) \dot{\mathrm{u}}_{i}(0)+I_{j k} \phi_{j m}(0) \dot{\phi}_{k m}(0)\right] d V+\int_{0}^{t} \int_{D} \rho \eta(0) \theta(\tau) d V d \tau+ \\
\quad+\int_{0}^{t} \int_{D}\left[\rho \dot{\mathrm{u}}_{i}(t) \dot{\mathrm{u}}_{i}(t)+I_{j k} \dot{\phi}_{j m}(t) \dot{\phi}_{k m}(t)-2 \Psi(\tau)-c \theta^{2}(\tau)\right] d V d \tau,
\end{gathered}
$$

for all $t \in[0, \infty)$.

Proof. We will consider the constitutive relations (4), the geometric Equation (2), and the motion Equations (9a) and (9b), we deduce

$$
\begin{aligned}
\frac{\partial}{\partial t}\left(\rho \mathrm{u}_{i}(t)\right. & \left.\dot{\mathrm{u}}_{i}(t)+I_{j k} \phi_{j m}(t) \dot{\phi}_{k m}(t)\right)= \\
= & \rho \dot{\mathrm{u}}_{i}(t) \dot{\mathrm{u}}_{i}(t)+I_{j k} \dot{\phi}_{j m}(t) \dot{\phi}_{k m}(t)+ \\
+ & {\left[\left(\tau_{i j}(t)+\eta_{i j}(t)\right) \mathrm{u}_{j}(t)+\mu_{i j k}(t) \phi_{j k}(t)\right]_{, i}-} \\
& \quad-\left[\left(\tau_{i j}(t)+\eta_{i j}(t)\right) \varepsilon_{i j}(t)+\eta_{i j}(t) \kappa_{i j}(t)+\mu_{i j k}(t) \chi_{i j k}(t)\right] .
\end{aligned}
$$

Taking into account definition (13), the last parentheses from the right-hand side of (29) can be restated in the following form:

$$
\begin{aligned}
\tau_{i j}(t) \varepsilon_{i j}(t)+\eta_{i j}(t) \kappa_{i j}(t)+\mu_{i j k}(t) \chi_{i j k}(t)= & \\
= & \left(\frac{1}{2} c \theta^{2}(t)+\Psi(t)\right)+\left(\frac{1}{T_{0}} \theta(t) \int_{0}^{t} q_{i}(\tau) d \tau\right)_{, i}- \\
& -\frac{1}{T_{0}} K_{i j}\left(\int_{0}^{t} \theta(\tau) d \tau\right)_{, i}\left(\int_{0}^{t} \theta(\tau) d \tau\right)_{, i}-\rho \eta(0) \theta(t) .
\end{aligned}
$$

Here, the expression of the entropy $\eta$ is obtained by integrating its equation of evolution (19), with regards to time variable. 
We integrate the equality (30) on $[0, t] \times D$, and take into account the data on the border (17) and the theorem of divergence. As such, we arrive at the proposed equality (29), and the demonstration of proposition is over.

Proposition 4. Let us consider a solution $\left(\mathrm{u}_{i}, \phi_{i j}, \theta\right)$ of the mixed problem $\mathcal{P}^{\prime}$. Then, the following equality takes place

$$
\begin{gathered}
2 \int_{D}\left[\rho \mathrm{u}_{i}(t) \dot{\mathrm{u}}_{i}(t)+I_{j k} \phi_{j m}(t) \dot{\phi}_{k m}(t)-\frac{1}{2 T_{0}} K_{i j}\left(\int_{0}^{t} \theta(\tau) d \tau\right)_{, i}\left(\int_{0}^{t} \theta(\tau) d \tau\right){ }_{, j}\right] d V \\
=\int_{D}\left[\rho \dot{\mathrm{u}}_{i}(0) \mathrm{u}_{i}(2 t)+I_{j k} \dot{\phi}_{j m}(0) \phi_{k m}(2 t)\right] d V+ \\
+\int_{D}\left[\rho \mathrm{u}_{i}(0) \dot{\mathrm{u}}_{i}(2 t)+I_{j k} \phi_{j m}(0) \dot{\phi}_{k m}(2 t)\right] d V- \\
\quad-\int_{0}^{t} \int_{D} \rho \eta(0)[\theta(t+\tau)-\theta(t-\tau)] d V d \tau,
\end{gathered}
$$

for all $t \in[0, \infty)$.

Proof. We will take into account the geometric relations (2) and the motion Equations (9a) and (9b), we deduce the following identity

$$
\begin{gathered}
\frac{\partial}{\partial t}\left[\rho\left(\dot{\mathrm{u}}_{i}(t+\tau) \mathrm{u}_{i}(t-\tau)+\mathrm{u}_{i}(t+\tau) \dot{\mathrm{u}}_{i}(t-\tau)\right)\right]+ \\
+\frac{\partial}{\partial t}\left[I_{j k}\left(\dot{\phi}_{j m}(t+\tau) \phi_{k m}(t-\tau)+\phi_{j m}(t+\tau) \dot{\phi}_{k m}(t-\tau)\right)\right]= \\
=\left[\left(\tau_{i j}+\eta_{i j}\right)(t+\tau) \mathrm{u}_{i}(t-\tau)-\left(\tau_{i j}+\eta_{i j}\right)(t-\tau) \mathrm{u}_{j}(t+\tau)+\right. \\
\left.\quad+\mu_{i j k}(t+\tau) \phi_{j k}(t-\tau)-\mu_{i j k}(t-\tau) \phi_{j k}(t+\tau)\right]_{, i}- \\
-\left[\left(\tau_{i j}+\eta_{i j}\right)(t+\tau) \varepsilon_{i j}(t-\tau)-\left(\tau_{i j}+\eta_{i j}\right)(t-\tau) \varepsilon_{i j}(t+\tau)\right]- \\
-\left[\mu_{i j k}(t+\tau) \chi_{i j k}(t-\tau)-\mu_{i j k}(t-\tau) \chi_{j k}(t+\tau)\right] .
\end{gathered}
$$

On the other hand, by using the symmetry Equation (8) and the constitutive relations (4), the last two brackets receive the following form:

$$
\begin{gathered}
{\left[\left(\tau_{i j}+\eta_{i j}\right)(t+\tau) \varepsilon_{i j}(t-\tau)-\left(\tau_{i j}+\eta_{i j}\right)(t-\tau) \varepsilon_{i j}(t+\tau)\right]+} \\
+\left[\mu_{i j k}(t+\tau) \chi_{i j k}(t-\tau)-\mu_{i j k}(t-\tau) \chi_{j k}(t+\tau)\right]= \\
=\frac{1}{T_{0}}\left[\theta(t+\tau) \int_{0}^{t-\tau} q_{i}(s) d s-\theta(t-\tau) \int_{0}^{t+\tau} q_{i}(s) d s\right]_{, i}- \\
-\frac{1}{T_{0}} K_{i j}\left[\left(\int_{0}^{t+\tau} \theta(s) d s\right)_{, i}\left(\int_{0}^{t-\tau} \theta(s) d s\right)_{, j}-\left(\int_{0}^{t+\tau} \theta(s) d s\right)_{, i}\left(\int_{0}^{t-\tau} \theta(s) d s\right)_{, j}\right]- \\
-\rho \eta(0)[\theta(t+\tau)-\theta(t-\tau)] .
\end{gathered}
$$

Here, the expression of the entropy $\eta$ is obtained by integrating its equation of evolution (19), with regards to time variable.

Let us integrate the equality (33) on $[0, t] \times D$, and take into account the border data (17) and the theorem of divergence. As such, we arrive at the proposed equality (31) and the demonstration of the proposition is over. 
If we combine the results from Equations (25) and (31), then we obtain a new useful equality

$$
\begin{aligned}
2 \int_{B}\left[\left(\rho \dot{\mathrm{u}}_{i}(t) \dot{\mathrm{u}}_{i}(t)+I_{j k} \dot{\phi}_{j m}(t) \dot{\phi}_{k m}(t)\right)-c \theta^{2}(t)\right] d V= \\
=-2 \int_{D}\left[\rho \mathrm{u}_{i}(0) \dot{\mathrm{u}}_{i}(0)+I_{j k} \phi_{j m}(0) \dot{\phi}_{k m}(0)\right] d V+ \\
\quad+\int_{D}\left[\rho \dot{\mathrm{u}}_{i}(0) \mathrm{u}_{i}(2 t)+I_{j k} \dot{\phi}_{j m}(0) \phi_{k m}(2 t)\right] d V+ \\
\quad+\int_{D}\left[\rho \mathrm{u}_{i}(0) \dot{\mathrm{u}}_{i}(2 t)+I_{j k} \phi_{j m}(0) \dot{\phi}_{k m}(2 t)\right] d V+ \\
\quad-\int_{0}^{t} \int_{D} \rho \eta(0)[2 \theta(\tau)+\theta(t+\tau)-\theta(t-\tau)] d V d \tau
\end{aligned}
$$

for all $t \in[0, \infty)$.

Based on the previously demonstrated integral identities, we are able to address the main results of our study. First, we established a result of uniqueness for the solution of the backward in time problem. As a consequence, we approach the question of localization of the solutions of the backward in time problem.

Theorem 1. At most, an ordered array $\mathbf{u}=\left(\mathrm{u}_{i}, \phi_{i j}, \theta\right)$ can satisfy the equations and conditions of the backward problem $\mathcal{P}^{\prime}$.

Proof. As usual, we will assume, by absurdum, that the problem would admit two solutions. The difference of the two solutions is also a solution, because the problem $\mathcal{P}^{\prime}$ is a linear one. Suffice it to show that this difference is null. For this we have to show that the problem $\mathcal{P}^{\prime}$, for which the boundary and initial data are null, admits the null solution. It is clear that for the difference of two solutions, the boundary and initial conditions become homogeneous.

To simplify writing, we introduce the function $M$ as a measure of the solution, defined by

$$
M(t)=\int_{D}\left[\frac{\varepsilon}{2}\left(\rho \dot{\mathrm{u}}_{i}(t) \dot{\mathrm{u}}_{i}(t)+I_{j k} \dot{\phi}_{j m}(t) \dot{\phi}_{k m}(t)\right)+(\varepsilon+2) \Psi(t)+\frac{\varepsilon}{2} c \theta^{2}(t)\right] .
$$

Here, $\varepsilon$ is a small positive number.

Based on assumptions (a), (b), and (c), it can be deduced that the function $M$ is positive.

Because the initial data are zero, the identity (18) received the simpler form:

$$
\begin{aligned}
& \int_{B}\left[\frac{1}{2}\left(\rho \dot{\mathrm{u}}_{i}(t) \dot{\mathrm{u}}_{i}(t)+I_{j k} \dot{\phi}_{j m}(t) \dot{\phi}_{k m}(t)\right)+\Psi(t)+\frac{1}{2} c \theta^{2}(t)\right] d V= \\
& =\int_{0}^{t} \int_{D} \frac{1}{T_{0}} K_{i j} \theta_{, i}(\tau) \theta_{, j}(\tau) d V d \tau, \forall t \in[0, \infty) .
\end{aligned}
$$

Analogously, the identity (21) becomes

$$
\begin{gathered}
\int_{B}\left[\frac{1}{2}\left(\rho \dot{\mathrm{u}}_{i}(t) \dot{\mathrm{u}}_{i}(t)+I_{j k} \dot{\phi}_{j m}(t) \dot{\phi}_{k m}(t)\right)+\Psi(t)-\frac{1}{2} c \theta^{2}(t)\right] d V= \\
=-\int_{0}^{t} \int_{D}\left\{\dot{\mathrm{u}}_{i}(\tau)\left[\left(a_{i j}+b_{j i}\right) \theta(\tau)\right]_{, j}+\dot{\phi}_{i j}(\tau)\left[c_{i j k} \theta(\tau)\right]_{, k}-\right. \\
\left.\quad-b_{i j} \dot{\phi}_{i j}(\tau) \theta(\tau)+\frac{1}{T_{0}} K_{i j} \theta_{, i}(\tau) \theta_{, j}(\tau)\right\} d V d \tau, \forall t \in[0, \infty) .
\end{gathered}
$$

If we use Equations (36) and (37), then the function $M$ from (35) becomes

$$
\begin{aligned}
& M(t)=-\int_{0}^{t} \int_{D}\left\{2 \dot{\mathrm{u}}_{i}(\tau)\left[\left(a_{i j}+b_{j i}\right) \theta(\tau)\right]_{, j}+\dot{\phi}_{i j}(\tau)\left[c_{i j k} \theta(\tau)\right]_{, k}-\right. \\
& \left.-b_{i j} \dot{\phi}_{i j}(\tau) \theta(\tau)+\frac{1-\varepsilon}{T_{0}} K_{i j} \theta_{, i}(\tau) \theta_{, j}(\tau)\right\} d V d \tau, \forall t \in[0, \infty) .
\end{aligned}
$$


By direct derivation with regards to the variable $t$ in (37), we are led to the following equality:

$$
\begin{aligned}
& \frac{d M(t)}{d t}=-2 \int_{D}\left\{\dot{\mathrm{u}}_{i}(\tau)\left[\left(a_{i j}+b_{j i}\right) \theta(\tau)\right]_{, j}+\dot{\phi}_{i j}(\tau)\left[c_{i j k} \theta(\tau)\right]_{, k}-\right. \\
& \left.-b_{i j} \dot{\phi}_{i j}(\tau) \theta(\tau)+\frac{1-\varepsilon}{2 T_{0}} K_{i j} \theta_{, i}(\tau) \theta_{, j}(\tau)\right\} d V, \forall t \in[0, \infty) .
\end{aligned}
$$

With the help of Schwarz' inequality and by using the arithmetic-geometric mean inequality, from (39) we can deduce the following inequality:

$$
\begin{aligned}
& \frac{d M(t)}{d t} \leq C_{1} \int_{D}\left[\rho \dot{\mathrm{u}}_{i}(t) \dot{\mathrm{u}}_{i}(t)+I_{j k} \dot{\phi}_{j m}(t) \dot{\phi}_{k m}(t)+c \theta^{2}(t)\right] d V+ \\
& +\frac{\delta-1+\mathcal{\varepsilon}}{T_{0}} \int_{D} K_{i j} \theta_{, i}(\tau) \theta_{, j}(\tau) d V, \forall t \in[0, \infty) .
\end{aligned}
$$

Now, we take into account that the internal energy density $\Psi$ is a positive definite quadratic form, according to the hypothesis (c), using the definition (35) of the function $M$ and choose $\delta \leq 1-\varepsilon$. Then, from (40) we obtain the inequality:

$$
\frac{d M(t)}{d t} \leq \frac{C_{1}}{\varepsilon} \int_{D} \varepsilon\left[\rho \dot{\mathrm{u}}_{i}(t) \dot{\mathrm{u}}_{i}(t)+I_{j k} \dot{\phi}_{j m}(t) \dot{\phi}_{k m}(t)+c \theta^{2}(t)\right] d V \leq \frac{C_{1}}{\varepsilon} M,
$$

and it is then clear that a solution to this inequality meets the next inequality:

$$
0 \leq M(t) \leq M(0) e^{C_{1} / \varepsilon}
$$

We recall that for the difference of the two supposed solutions, the initial conditions are homogeneous, then we have $M(0)=0$, so from (42) we get

$$
M(t)=0, \forall t \in[0, \infty)
$$

and this together with the assumptions leads to the conclusion that our problem has only the solution

$$
\mathrm{u}_{i}(t)=0, \phi_{i j}(t)=0, \theta(t)=0, \forall t \in[0, \infty),
$$

and the proof of Theorem 1 is concluded.

Our final result is dedicated to the partition of various energies associated with the solution of the backward in time problem $\mathcal{P}^{*}$. We recall that this problem consists of the equations of motion (9), the constitutive relations (4), the kinematic Equation (2), the initial data (12), and the boundary restrictions in their homogeneous form (11).

First, using the procedure outlined at the end of Section 2, we transform the boundary-final value problem $\mathcal{P}^{*}$ into the boundary-initial value problem $\mathcal{P}^{\prime}$. In this way, in what follows, we will be able to make the considerations only on the problem $\mathcal{P}^{\prime}$. Let us denote by $\mathcal{T}$ the set of of those thermoelastodynamic processes defined in the cylinder $(-\infty, 0] \times D$, which satisfy the restriction

$$
\int_{D} \frac{1}{T_{0}} K_{i j} \theta_{, i}(\tau) \theta_{, j}(\tau) d V \leq C_{1}
$$

for all $t \in[0, \infty)$. Here, $C_{1}$ is a given positive constant. 
We will introduce the known Cesaro means necessary to evaluate the various types of energies that can be attached to a solution to the problem $\mathcal{P}^{\prime}$. So, if $\left(\mathrm{u}_{i}, \phi_{i j}, \theta\right)$ is a solution of the mixed problem, then the Cesaro means are:

$$
\begin{gathered}
K(t)=\frac{1}{t} \int_{0}^{t} \int_{D}\left[\rho \dot{\mathrm{u}}_{i}(\tau) \dot{\mathrm{u}}_{i}(\tau)+I_{j k} \dot{\phi}_{j m}(\tau) \dot{\phi}_{j m}(\tau)\right] d V d \tau \\
S(t)=\frac{1}{t} \int_{0}^{t} \int_{D}\left[\frac{1}{2} A_{i j m n} \varepsilon_{i j}(\tau) \varepsilon_{m n}(\tau)+D_{i j m n} \varepsilon_{i j}(\tau) \kappa_{m n}(\tau)+F_{i j m n r} \varepsilon_{i j}(\tau) \chi_{m n r}(\tau)+\right. \\
\left.\quad+\frac{1}{2} B_{i j m n} \kappa_{i j}(\tau) \kappa_{m n}(\tau)+G_{i j m n r} \kappa_{i j}(\tau) \chi_{m n r}(\tau)+\frac{1}{2} C_{i j k m n r} \chi_{i j k}(\tau) \chi_{m n r}(\tau)\right] d V d \tau ; \\
R(t)=\frac{1}{t} \int_{0}^{t} \int_{D} \frac{1}{2} c \theta^{2}(\tau) d V d \tau ; \\
D(t)=\frac{1}{t} \int_{0}^{t} \int_{0}^{\tau} \int_{D} \frac{1}{T_{0}} K_{i j} \theta_{, i}(s) \theta_{, j}(s) d V d s d \tau .
\end{gathered}
$$

In the particular case when meas $\left(\partial D_{u}\right)=0$ and meas $\left(\partial D_{\phi}\right)=0$, it can be determined a rigid displacement, a rigid dipolar displacement, and a null temperature, which satisfy the equations of motion (9), the constitutive relations (4), the kinematic relations (2), and verify the homogeneous boundary conditions (17). In this case, the initial data can be decomposed as follows:

$$
\begin{aligned}
& \mathrm{u}_{i}^{0}=\mathrm{u}_{i}^{\prime}+V_{i}^{0}, \dot{\mathrm{u}}_{i}^{0}=\dot{\mathrm{u}}_{i}^{\prime}+\dot{V}_{i}^{0}, \\
& \phi_{i j}^{0}=\phi_{i j}^{\prime}+\Psi_{i j}^{0} \dot{\phi}_{i j}^{0}=\dot{\phi}_{i j}^{\prime}+\dot{\Psi}_{i j}^{0} .
\end{aligned}
$$

The rigid displacements $\mathrm{u}_{i}^{\prime}, \dot{\mathrm{u}}_{i}^{\prime}, \phi_{i j}^{\prime}, \dot{\phi}_{i j}^{\prime}$ can be computed using the functions

$$
W_{i}(\omega)=\int_{D} \rho \omega_{i} D v, W_{j k}(\psi)=\int_{D} I_{m n} \psi_{m j} \psi_{n k} d V,
$$

such that we have

$$
\begin{aligned}
& W_{i}\left(V_{i}^{0}\right)=0, W_{i}\left(\dot{V}_{i}^{0}\right)=0, \\
& W_{j k}\left(\Psi_{i j}^{0}\right)=0, W_{j k}\left(\dot{\Psi}_{i j}^{0}\right)=0 .
\end{aligned}
$$

Together with known notation $C^{1}(D)$, we will use the notation $W_{n}(D)$ for a Sobolev space defined on the domain $\mathrm{D}$, and $\mathbf{W}_{n}(D)=\left[W_{n}(D)\right]^{3}$.

Other new notations:

$$
\begin{aligned}
& \mathcal{C}^{1}(D)=\left\{\left(\mathrm{u}_{i}, \phi_{i j}\right) \in C^{1}(D)^{3} \times C^{1}(D)^{9}: \mathrm{u}_{i}=0 \text { on } \partial D_{\mathrm{u}}, \phi_{i j}=0 \text { on } \partial D_{\phi} ;\right. \\
& \text { if meas } \left.\left(\partial D_{u}\right)=0 \text { and meas }\left(\partial D_{\phi}\right)=0, \text { then } W_{i}\left(\mathrm{u}_{i}\right)=0, W_{j k}\left(\phi_{i j}^{0}\right)=0\right\} ; \\
& \tilde{C}^{1}(D)=\left\{\theta \in C^{1}(D): \theta=0 \text { on } \partial D_{\theta}\right\} ; \\
& \mathcal{W}_{1}(D) \text { the completion of } \mathcal{C}^{1}(D) ; \\
& \tilde{W}_{1}(D) \text { the completion of } \tilde{C}^{1}(D),
\end{aligned}
$$

the completion is by means of the original norm of the respective Sobolev space. Based on definition (13), and taking into account the hypothesis (15), we can obtain the following inequality (see [34]):

$$
\int_{D} \Psi(\mathbf{u}) d V \geq \frac{M_{1}}{2} \int_{D}\left[\rho \mathrm{u}_{i} \mathrm{u}_{i}+I_{j k} \phi_{j m} \phi_{k m}\right] d V,
$$

for any $\mathbf{u}=\left(\mathrm{u}_{i}, \phi_{i j}\right) \in \mathcal{W}_{1}(D) . M_{1}>0$ is a convenient chosen constant. 
On the other hand, if we take into account the hypothesis (14), we can obtain the next inequality of Poincare' type:

$$
\int_{D} K_{i j} \theta_{, i} \theta_{, j} d V \geq M_{2} \int_{D} \theta^{2} d V
$$

for any $\theta \in \tilde{W}_{1}(D) . M_{2}>0$ is a convenient chosen constant.

Let us consider a solution $\left(\mathrm{u}_{i}, \phi_{i j}, \theta\right)$ to the problem $\mathcal{P}^{\prime}$ in the particular case when meas $\left(\partial D_{u}\right)=0$ and meas $\left(\partial D_{\phi}\right)=0$. We can represent this solution in the following form:

$$
\begin{aligned}
& \mathrm{u}_{i}(t, x)=u_{i}(t, x)+u_{i}^{\prime}(t, x)+t \dot{u}_{i}^{\prime}(t, x), \theta(t, x)=\vartheta(t, x), \\
& \phi_{i j}(t, x)=\psi_{i j}(t, x)+\phi_{i j}^{\prime}(t, x)+t \dot{\phi}_{i j}^{\prime}(t, x),(t, x) \in[0, \infty) \times D,
\end{aligned}
$$

in which $\left(u_{i}, \psi_{i j}, \vartheta\right) \in \mathcal{W}_{1}(D) \times \tilde{W}_{1}(D)$. In addition, the ordered array $\left(u_{i}, \psi_{i j}, \vartheta\right)$ satisfies the problem $\mathcal{P}^{\prime}$, which corresponds to the following initial data:

$$
\begin{aligned}
& u_{i}(0, x)=V_{i}(x), \dot{u}_{i}(0, x)=\dot{V}_{i}(x), \theta(t, x)=\vartheta(x), \\
& \phi_{i j}(0, x)=\Psi_{i j}(x), \dot{\phi}_{i j}(0, x)=\dot{\Psi}_{i j}(x), \forall x \in D .
\end{aligned}
$$

We now have everything prepared to address the problem of the equipartition of the various types of energies associated with the solution to the problem $\mathcal{P}^{\prime}$.

Theorem 2. Consider a solution $\left(\mathrm{u}_{i}, \phi_{i j}, \theta\right)$ of the backward in time problem $\mathcal{P}^{\prime}$. If the initial data satisfy the following conditions:

$$
\begin{aligned}
& \mathbf{u}=\left(\mathrm{u}_{i}\right) \in \mathbf{W}_{1}(D), \dot{\mathbf{u}}=\left(\dot{\mathrm{u}}_{i}\right) \in \mathbf{W}_{0}(D), \\
& \quad \boldsymbol{\phi}=\left(\phi_{i j}\right) \in \mathbf{W}_{1}(D), \dot{\boldsymbol{\phi}}=\left(\dot{\phi}_{i j}\right) \in \mathbf{W}_{0}(D), \theta \in W_{0}(D),
\end{aligned}
$$

then the following three statements are true:

i) The thermal component of energy, $R$, vanishes as $t \rightarrow \infty$ :

$$
\lim _{t \rightarrow \infty} R(t)=0
$$

ii) if meas $\left(\partial D_{u}\right)=0$ and meas $\left(\partial D_{\phi}\right)=0$, then we have

$$
\begin{aligned}
& \lim _{t \rightarrow \infty} K(t)=\lim _{t \rightarrow \infty} S(t)+\frac{1}{2} \int_{D}\left[\rho \dot{\mathrm{u}}_{i}^{\prime} \dot{\mathrm{u}}_{i}^{\prime}+I_{j k} \dot{\phi}_{j m}^{\prime} \dot{\phi}_{k m}^{\prime}\right] d V \\
& \lim _{t \rightarrow \infty} D(t)=2 \lim _{t \rightarrow \infty} K(t)-\frac{1}{2} \int_{D}\left[\rho \dot{\mathrm{u}}_{i}^{\prime} \dot{\mathrm{u}}_{i}^{\prime}+I_{j k} \dot{\phi}_{j m}^{\prime} \dot{\phi}_{k m}^{\prime}\right] d V-E(0)= \\
& =2 \lim _{t \rightarrow \infty} S(t)+\frac{1}{2} \int_{D}\left[\rho \dot{\mathrm{u}}_{i}^{\prime} \dot{\mathrm{u}}_{i}^{\prime}+I_{j k} \dot{\phi}_{j m}^{\prime} \dot{\phi}_{k m}^{\prime}\right] d V-E(0) ;
\end{aligned}
$$

iii) if meas $\left(\partial D_{u}\right) \neq 0$ or meas $\left(\partial D_{\phi}\right) \neq 0$, then we have

$$
\begin{aligned}
& \lim _{t \rightarrow \infty} K(t)=\lim _{t \rightarrow \infty} S(t), \\
& \lim _{t \rightarrow \infty} D(t)=2 \lim _{t \rightarrow \infty} K(t)-E(0)= \\
& =2 \lim _{t \rightarrow \infty} S(t)-E(0),
\end{aligned}
$$

where, to simplify writing in (52) and (53), we used the notation:

$$
E(t)=\int_{D}\left[\frac{1}{2}\left(\rho \dot{\mathrm{u}}_{i}(t) \dot{\mathrm{u}}_{i}(t)+I_{j k} \dot{\phi}_{j m}(t) \dot{\phi}_{k m}(t)\right)+\Psi(t)+\frac{1}{2} c \theta^{2}(t)\right] d V,
$$


the internal energy density $\Psi$ being defined in (13).

Proof. Taking into account the relations (18), (54), and (44) we immediately deduce the following equality:

$$
K(t)+S(t)+R(t)=D(t)+E(0), \forall t \in(0, \infty) .
$$

Now, we consider the restrictions (43) and (48), the definitions (44), and the notation (54) in order to deduce the following estimation:

$$
R(t) \leq \frac{T_{0} C_{1}}{2 M_{2} t} \max _{x \in D}\{c(x)\}, \forall t \in(0, \infty)
$$

Clearly, since $c(x)$ is a continuous function, we deduce that $\max _{x \in D}\{c(x)\}$ is bounded, such that after we pass to the limit in (56) for $t \rightarrow \infty$, we obtain the result (50).

If we consider the identity (34) and use the notations (44), we are led to the the following identity:

$$
\begin{aligned}
& K(t)-S(t)-R(t)= \\
& =-\frac{1}{2 t} \int_{D}\left[\rho \mathrm{u}_{i}(0) \dot{\mathrm{u}}_{i}(0)+I_{j k} \phi_{j m}(0) \dot{\phi}_{k m}(0)\right] d V+ \\
& \quad+\frac{1}{4 t} \int_{D}\left[\rho \dot{\mathrm{u}}_{i}(0) \mathrm{u}_{i}(2 t)+I_{j k} \dot{\phi}_{j m}(0) \phi_{k m}(2 t)\right] d V+ \\
& \quad+\frac{1}{4 t} \int_{D}\left[\rho \mathrm{u}_{i}(0) \dot{\mathrm{u}}_{i}(2 t)+I_{j k} \phi_{j m}(0) \dot{\phi}_{k m}(2 t)\right] d V+ \\
& \quad-\frac{1}{4 t} \int_{0}^{t} \int_{D} \rho \eta(0)[2 \theta(\tau)+\theta(t+\tau)-\theta(t-\tau)] d V d \tau,
\end{aligned}
$$

for all $t \in[0, \infty)$.

On the other hand, taking into account the hypothesis (15), the inequalities (43) and (48), and the identity (18) we obtain the estimates

$$
\begin{aligned}
& \int_{D} \rho \dot{\mathrm{u}}_{i}(t) \dot{\mathrm{u}}_{i}(t) d V \leq 2\left(C_{1}+E(0)\right), \int_{D} I_{j k} \dot{\phi}_{j m}(t) \dot{\phi}_{k m}(t) d V \leq 2\left(C_{1}+E(0)\right), \\
& c_{0} \int_{D} \rho \theta^{2}(t) d V \leq 2\left(C_{1}+E(0)\right), \int_{D} 2 \Psi(t) d V \leq 2\left(C_{1}+E(0)\right),
\end{aligned}
$$

where $c_{0}$ is from hypothesis (a), $C_{1}$ is from (43), and $E$ from (54).

In (57) we use the Schwarz's inequality and taking into account the estimates (58), we pass the limit—for $t \rightarrow \infty$-so we get the equality

$$
\lim _{t \rightarrow \infty} K(t)=\lim _{t \rightarrow \infty} S(t)+\lim _{t \rightarrow \infty} \frac{1}{4 t} \int_{D}\left[\rho \dot{\mathrm{u}}_{i}(0) \mathrm{u}_{i}(2 t)+I_{j k} \dot{\phi}_{j m}(0) \dot{\phi}_{k m}(2 t)\right] d V .
$$

In this way, it will be easy to demonstrate the relation (53), if we show that the integral from the right-hand side of the identity (59) is bounded.

For this aim, we will use the fact that meas $\left(\partial D_{u}\right) \neq 0$ or meas $\left(\partial D_{\phi}\right) \neq 0$ and $\left(u_{i}, \psi_{i j}\right) \in \mathcal{W}_{1}(D)$. Furthermore, we consider the relations (47), (54), and (18) in order to get the following estimates:

$$
\begin{aligned}
& M_{1} \int_{D} \rho \mathrm{u}_{i}(t) \mathrm{u}_{i}(t) d V \leq \int_{D} 2 \Psi(t) d V \leq 2\left(C_{1}+E(0)\right), \\
& M_{1} \int_{D} I_{j k} \phi_{j m}(t) \phi_{k m}(t) d V \leq \int_{D} 2 \Psi(t) d V \leq 2\left(C_{1}+E(0)\right),
\end{aligned}
$$


such that it is easy, after we apply the Schwarz's inequality, to deduce

$$
\lim _{t \rightarrow \infty} \frac{1}{4 t} \int_{D}\left[\rho \dot{\mathrm{u}}_{i}(0) \mathrm{u}_{i}(2 t)+I_{j k} \dot{\phi}_{j m}(0) \phi_{k m}(2 t)\right] d V=0 .
$$

From (59) and (60) we obtain the first result from (53), and after we consider the equality (55), the second equality from (53) is proven.

Finally, we will prove the equalities (51) and (52). In this regard, we take into account that meas $\left(\partial D_{u}\right)=0$ and meas $\left(\partial D_{\phi}\right) \neq 0$ such that with the help of the decompositions (45) and (49), and conditions (46), we are led to the equality

$$
\begin{gathered}
\frac{1}{4 t} \int_{D}\left[\rho \dot{\mathrm{u}}_{i}(0) \mathrm{u}_{i}(2 t)+I_{j k} \dot{\phi}_{j m}(0) \phi_{k m}(2 t)\right] d V= \\
=\frac{1}{4 t} \int_{D} \rho \dot{\mathrm{u}}_{i}^{\prime} \mathrm{u}_{i}^{\prime}(2 t) d V+\frac{1}{4 t} \int_{D} \rho\left(\dot{\mathrm{u}}_{i}^{\prime}+\dot{V}_{i}^{0}\right) u_{i}(2 t) d V+ \\
\quad+\frac{1}{2} \int_{D} \rho \dot{\mathrm{u}}_{i}^{\prime} \dot{\mathrm{u}}_{i}^{\prime} d V+\frac{1}{4 t} \int_{D} I_{j k} \dot{\phi}_{j m}^{\prime} \phi_{k m}^{\prime} d V+ \\
+\frac{1}{4 t} \int_{D} I_{j k}\left(\dot{\phi}_{j m}^{\prime}+\dot{\Psi}_{j m}^{0}\right) \psi_{j k}(2 t) d V+\frac{1}{2} \int_{D} I_{j k} \dot{\phi}_{j m}^{\prime} \dot{\phi}_{k m}^{\prime} d V .
\end{gathered}
$$

Let us observe that relations (47), (54), and (18) involve the estimates

$$
\begin{aligned}
& M_{1} \int_{D} \rho u_{i}(t) u_{i}(t) d V \leq 2\left(C_{1}+E(0)\right), \\
& M_{1} \int_{D} I_{j k} \psi_{j m}(t) \psi_{k m}(t) d V \leq 2\left(C_{1}+E(0)\right),
\end{aligned}
$$

such that from (61) we are led to the equality

$$
\begin{aligned}
& \lim _{t \rightarrow \infty} \frac{1}{4 t} \int_{D}\left[\rho \dot{\mathrm{u}}_{i}(0) \mathrm{u}_{i}(2 t)+I_{j k} \dot{\phi}_{j m}(0) \phi_{k m}(2 t)\right] d V= \\
& =\frac{1}{2} \int_{D} \rho \dot{\mathrm{u}}_{i}^{\prime} \dot{\mathrm{u}}_{i}^{\prime} d V+\frac{1}{2} \int_{D} I_{j k} \dot{\phi}_{j m}^{\prime} \dot{\phi}_{k m}^{\prime} d V .
\end{aligned}
$$

Now, we substitute (62) into Equation (59) and then obtain the relation (51). Lastly, we consider the relations (50), (51), and (55) in order to obtain (52). With this, the proof of the theorem is completed.

\section{Conclusions}

We first formulate the mixed backward in time problem in the context of thermoelasticity for dipolar materials. To prove the consistency of this mixed problem, our first main result is regarding the uniqueness of the solution for this problem. This is obtained based on some auxiliary results, namely, four integral identities. The second main result is regarding the temporal behavior of our thermoelastic body with dipolar structure. This behavior is studied by means of some relations on partition of various parts of the energy associated to the solution of the problem. After we introduce the Cesaro means for all parts of the total energy, we can evaluate the asymptotic partition of these parts. We have to say that the kinetic energy and potential energy become asymptotically equal when the variable time tends to infinity.

Author Contributions: All four authors conceived the framework and structured the whole manuscript, checked the results and completed the revision of the paper. The authors have equally contributed to the elaboration of this manuscript.

Funding: This research received no external funding.

Acknowledgments: The authors would like to express their gratitude to the anonymous reviewers for their constructive comments and suggestions, which led to an improved form of the manuscript.

Conflicts of Interest: The authors declare no conflict of interest. 


\section{References}

1. Eringen, A.C. Theory of thermo-microstretch elastic solids. Int. J. Eng. Sci. 1990, 28, 1291-1301. [CrossRef]

2. Eringen, A.C. Microcontinuum Field Theories; Springer: New York, NY, USA, 1990

3. Hassan, M.; Marin, M.; Ellahi, R.; Alamri, S.Z. Exploration of convective heat transfer and flow characteristics synthesis by $\mathrm{Cu}-\mathrm{Ag} /$ water hybrid-nanofluids. Heat Transf. Res. 2018, 49, 1837-1848. [CrossRef]

4. Marin, M.; Ellahi, R.; Chirila, A. On solutions of Saint-Venant's problem for elastic dipolar bodies with voids. Carpathian J. Math. 2017, 33, 219-232.

5. Mindlin, R.D. Micro-structure in linear elasticity. Arch. Ration. Mech. Anal. 1964, 51-78. [CrossRef]

6. Green, A.E.; Rivlin, R.S. Multipolar continuum mechanics. Arch. Ration. Mech. Anal. 1964, 17, $113-147$. [CrossRef]

7. Fried, E.; Gurtin, M.E. Thermomechanics of the interface between a body and its environment. Contin. Mech. Therm. 2007, 19, 253-271. [CrossRef]

8. Nunziato, J.W.; Cowin, S.C. A nonlinear theory of materials with voids. Arch. Ration. Mech. Anal. 1979, 72, 175-201. [CrossRef]

9. Cowin, S.C.; Nunziato, J.W. Linear elastic materials with voids. J. Elast. 1983, 13, 125-147. [CrossRef]

10. Goodman, M.A.; Cowin, S.C. A continuum theory of granular material. Arch. Ration. Mech. Anal. 1971, 44, 249-266. [CrossRef]

11. Othman, M.I.A.; Marin, M. Effect of thermal loading due to laser pulse on thermo-elastic porous medium under G-N theory. Results Phys. 2017, 7, 3863-3872. [CrossRef]

12. Abbas, I.A. A GN model based upon two-temperature generalized thermoelastic theory in an unbounded medium with a spherical cavity. Appl. Math. Comput. 2014, 245, 108-115. [CrossRef]

13. Abbas, I.A. Eigenvalue approach for an unbounded medium with a spherical cavity based upon two-temperature generalized thermoelastic theory. J. Mech. Sci. Technol. 2014, 28, 4193-4198. [CrossRef]

14. Othman, M.I.A.; Hasona, W.M.; Abd-Elaziz, E.M. Effect of Rotation on Micropolar Generalized Thermoelasticity with Two-Temperatures using a Dual-Phase-Lag Model. Can. J. Phys. 2014, 92, 149-158. [CrossRef]

15. Marin, M. Cesaro means in thermoelasticity of dipolar bodies. Acta Mech. 1997, 122, 155-168. [CrossRef]

16. Marin, M.; Craciun, E.M. Uniqueness results for a boundary value problem in dipolar thermoelasticity to model composite materials. Compos. Part B Eng. 2017, 126, 27-37. [CrossRef]

17. Serrin, J. The Initial Value Problem for the Navier-Stokes Equations. In Nonlinear Problems Proc. Sympos.; Univ. of Wisconsin Press: Madison, WI, USA, 1963; pp. 69-98.

18. Ciarletta, M.; Scalia, A. Some Results in Linear Theory of Thermomicrostretch Elastic Solids. Meccanica 2004, 39, 191-206. [CrossRef]

19. Knops R.J.; Payne, L.E. On the Stability of Solutions of the Navier-Stokes Equations Backward in Time. Arch. Ration. Mech. Anal. 1968, 29, 331-335. [CrossRef]

20. Galdi, G.P.; Straughan, B. Stability of Solutions of the Navier-Stokes Equations Backward in Time. Arch. Ration. Mech. Anal. 1988, 101, 107-114. [CrossRef]

21. Payne, L.; Straughan, B. Improperly Posed and Nonstandard Problems for Parabolic Partial Differential Equations. In Elasticity: Mathematical Methods and Applications; Ellis-Horwood Pub: Chichester, UK, 1990; pp. 273-300.

22. Ames, K.A.; Payne, L.E. Stabilizing Solutions of the Equations of Dynamical Linear Thermoelasticity Backward in Time. Stab. Appl. Anal. Contin. Media 1991, 1, 243-260.

23. Ciarletta, M. On the Uniqueness and Continuous Dependence of Solutions in Dynamical Thermoelasticity Backward in Time. J. Therm. Stress. 2002, 25, 969-984. [CrossRef]

24. Ciarletta, M.; Chirita, S. Spatial Behavior in Linear Thermoelasticity Backward in Time. In Proceedings of the Fourth International Congress on Thermal Stresses, Osaka, Japan, 8-11 June 2001; pp. 485-488.

25. Quintanilla, R. Impossibility of Localization in Linear Thermoelasticity with Voids. Mech. Res. Commun. 2007, 34, 522-527. [CrossRef]

26. Green A.E.; Naghdi, P.M. On Undamped Heat Waves in an Elastic Solid. J. Therm. Stress. 1992, 15, $253-264$. [CrossRef]

27. Green A.E.; Naghdi, P.M. Thermoelasticity without Energy Dissipation. J. Elast. 1993, 31, 189-208. [CrossRef] 
28. Iovane, G.; Passarella, F. Saint-Venant's Principle in Dynamic Porous Thermoelastic Media with Memory for Heat Flux. J. Therm. Stress. 2004, 27, 983-999. [CrossRef]

29. Passarella, F.; Tibullo, V. Some Results in Linear Theory of Thermoelasticity Backward in Time for Microstretch Materials. J. Therm. Stress. 2010, 33, 559-576. [CrossRef]

30. Quintanilla, R.; Straughan, B. Energy Bounds for Some Non-standard Problems in Thermoelasticity. Proc. R. Soc. A Math. Phys. Eng. Sci. 2005, 461, 1-15. [CrossRef]

31. De Sciarra, F.M.; Salerno, M. On thermodynamic functions in thermoelasticity without energy dissipation. Eur. J. Mech. A Solids 2014, 46, 84-95. [CrossRef]

32. Canadija, M.; Barretta, R.; de Sciarra, F.M. A gradient elasticity model of Bernoulli-Euler nanobeams in non-isothermal environments. Eur. J. Mech. A Solids 2016, 55, 243-255. [CrossRef]

33. Barretta, R.; Canadija, M.; Luciano, R.; de Sciarra, F.M. Stress-driven modeling of nonlocal thermoelastic behavior of nanobeams. Int. J. Eng. Sci. 2018, 126, 53-67. [CrossRef]

34. Hlavacek, I.; Hlavacek, M. On the Existence and Uniqueness of Solution and Some Variational Principles in Linear Theories of Elasticity with Couple-Stresses. I. Cosserat Continuum. Appl. Math. 1969, 14, 387-410.

(c) 2019 by the authors. Licensee MDPI, Basel, Switzerland. This article is an open access article distributed under the terms and conditions of the Creative Commons Attribution (CC BY) license (http://creativecommons.org/licenses/by/4.0/). 